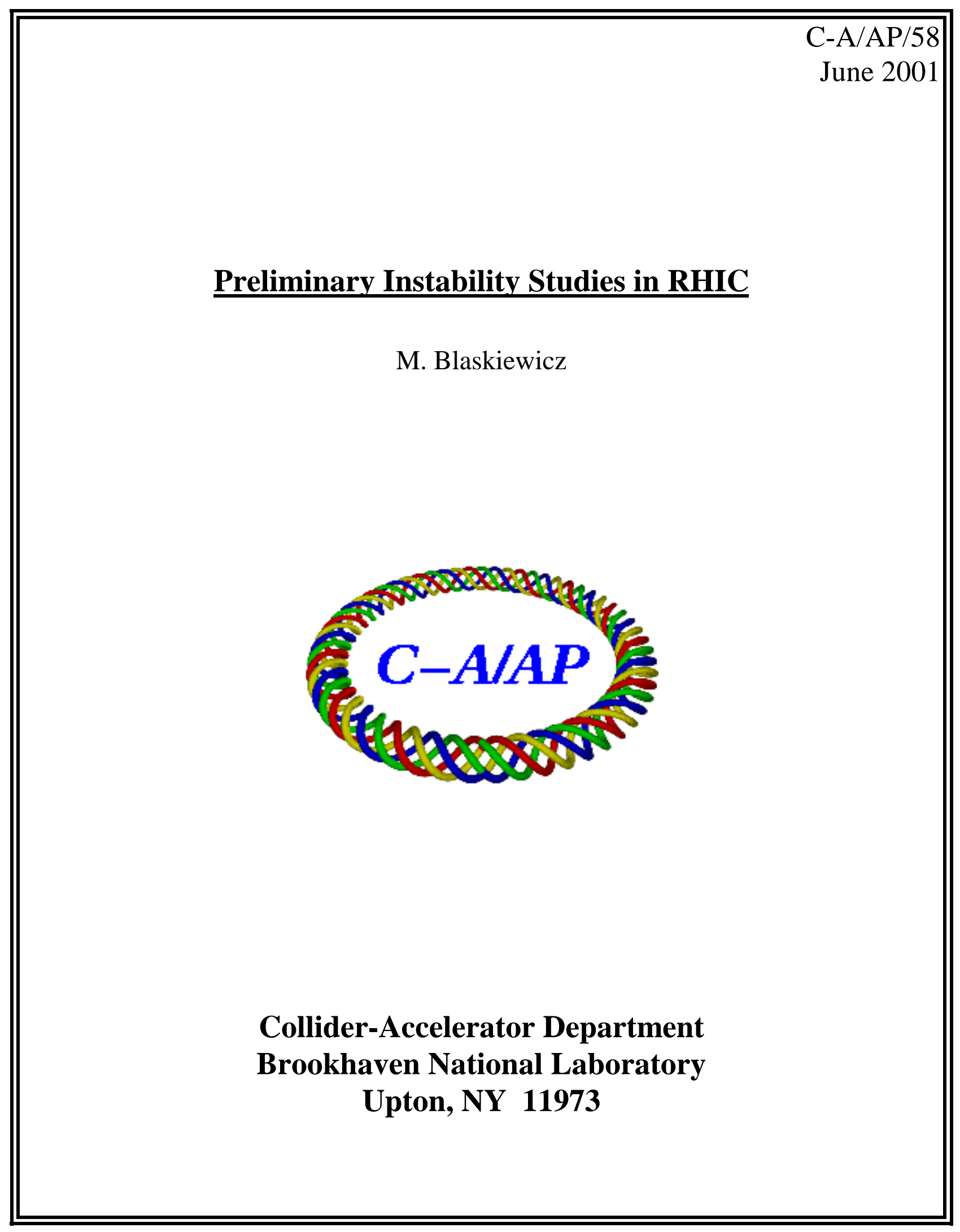




\title{
Preliminary Instability Studies in RHIC
}

\author{
M. Blaskiewicz
}

June 28, 2001

\section{Data}

A slow, single bunch, horizontal instability appeared on the RHIC injection porch with positive chromaticity. Data taken on 1 June 2001 are summarized in Table 1. The data for the growth rate are shown on the last page of this document.

Table 1

\begin{tabular}{|c|c|c|}
\hline parameter & symbol & value \\
\hline species & $Z, A$ & $\mathrm{Au} 79^{+}$ \\
\hline energy & $\gamma$ & 10.5 \\
\hline particles/bunch & $N_{b}$ & $1 \rightarrow 2 \times 10^{8}$ \\
\hline rms bunch length & $\sigma_{t}$ & $8.5 \mathrm{~ns}$ \\
\hline chromaticity & $Q_{\beta}^{\prime}$ & $-2 \rightarrow 2$ \\
\hline tune & $Q_{\beta}$ & 28.2 \\
\hline growth rate & $\operatorname{Im}\left(\omega_{\beta}\right)$ & $\approx 1.2 s^{-1}$ \\
\hline pipe radius & $b$ & $3.6 \mathrm{~cm}$ \\
\hline
\end{tabular}

The purpose of this note is to show that these data are consistent with the resistive wall impedance.

\section{Analysis}

The coherent betatron frequency in the weak coupling limit of the Wang formalism[1] is

$$
\Delta \omega_{m}=m \omega_{s}-i \frac{\bar{I} c}{4 \pi 2^{|m|}|m| !\left(A m_{p} / Z e\right) Q_{\beta}} \sum_{n=-\infty}^{\infty} Z_{\perp}\left[(n M+s) \omega_{0}+\omega_{\beta}\right] e^{-\tilde{n}^{2} \sigma^{2}}(\tilde{n} \sigma)^{2|m|},
$$

$m=\ldots,-1,0,1, \ldots$ is the synchrotron mode number, $\omega_{s}$ is the angular synchrotron frequency, $\bar{I}$ is the average current, $M$ is the number of bunches, $s$ is the coupled bunch mode number, $E_{T} / q$ is the total proton energy divided by its charge, $\sigma=\sigma_{t} / \omega_{0}$ is the rms bunch length in units of machine azimuth, $\tilde{n}=n M+s+Q_{\beta}-Q_{\beta}^{\prime} / \eta$, and $\eta=1 / \gamma_{T}^{2}-1 / \gamma^{2}$ is the slip factor. This is a standard formula that can be found in ZAP, Chao's book, etc.

For the purposes of this note take the transverse impedance to be give soley by the resistive wall impedance.

$$
Z_{\perp, r w}=\frac{2 \rho_{e} R c(1-i \operatorname{sgn}(\omega))}{\omega b^{3} \delta}
$$


The skin depth is given by

$$
\delta=\sqrt{\frac{2 \rho_{e}}{\mu_{0}|\omega|}}
$$

where $\rho_{e}$ is the electrical conductivity, $\mu_{0}$ is the vacuum permeability, and $\omega=2 \pi f$ is the angular frequency, with all quantities in MKS. For cold stainless steel the conductivity is $\rho_{e}=7.3 \times 10^{-7} \Omega \mathrm{m}[2]$, which is only slightly smaller than the room temperature value.

Taking one bunch $(M=1)$ with $N_{b}=10^{8}$ and the other parameters in Table 1 the growth rates are given in Table 2. Also given in the table is the number of ions per bunch for which the calculated and measured growth rates agree $N_{\text {fit }}$

Table 2

\begin{tabular}{|c|c|c|}
\hline$Q_{\beta}^{\prime}$ & $\operatorname{Im}\left(\omega_{\beta}\right) \mathrm{s}^{-1}$ & $N_{f i t}$ \\
\hline 1.0 & 0.44 & $2.7 \times 10^{8}$ \\
\hline 1.5 & 0.66 & $1.8 \times 10^{8}$ \\
\hline 2.0 & 0.86 & $1.4 \times 10^{8}$ \\
\hline
\end{tabular}

Given the uncertainties there is no reason to assume that the transverse impedance in RHIC is significantly larger than previously thought [3] A more stringent test is suggested in this figure.

$10^{\star *} 9$ ions/bunch

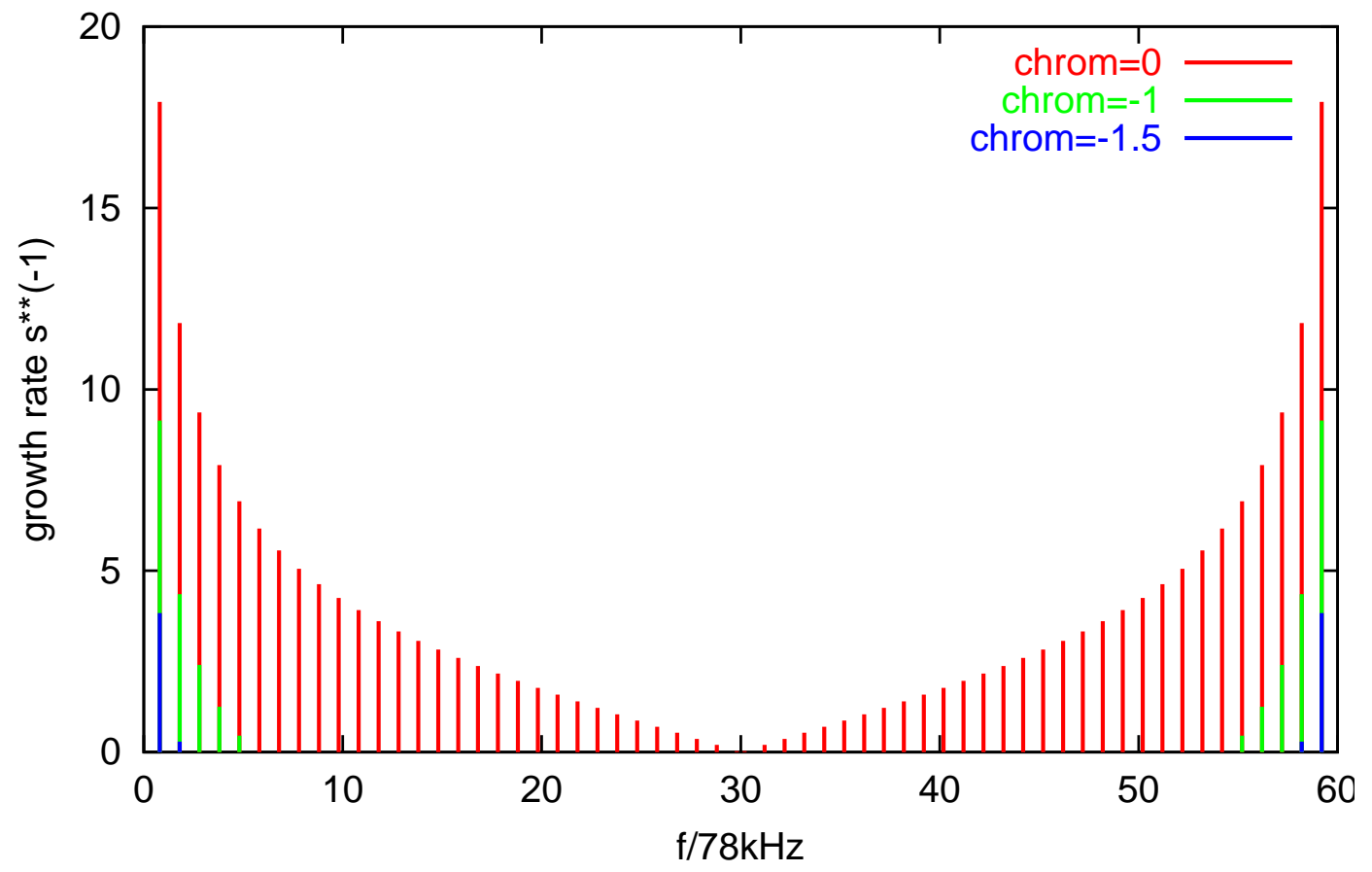

This shows the coupled bunch growth rates for 60 bunches and $10^{9}$ ions/bunch for various chromaticities, as a function of observed frequency. The frequency dependence of the growth rate is due to the frequency dependence of the resistive wall impedance. Experimental tests should be fairly easy. 


\section{References}

[1] J.M. Wang, SLAC summer school, (1985).

[2] Gary McIntyre, private communication.

[3] Collective Instabilities in RHIC, S. Peggs \& W.W. MacKay eds., RHIC/AP/36 (1994). 


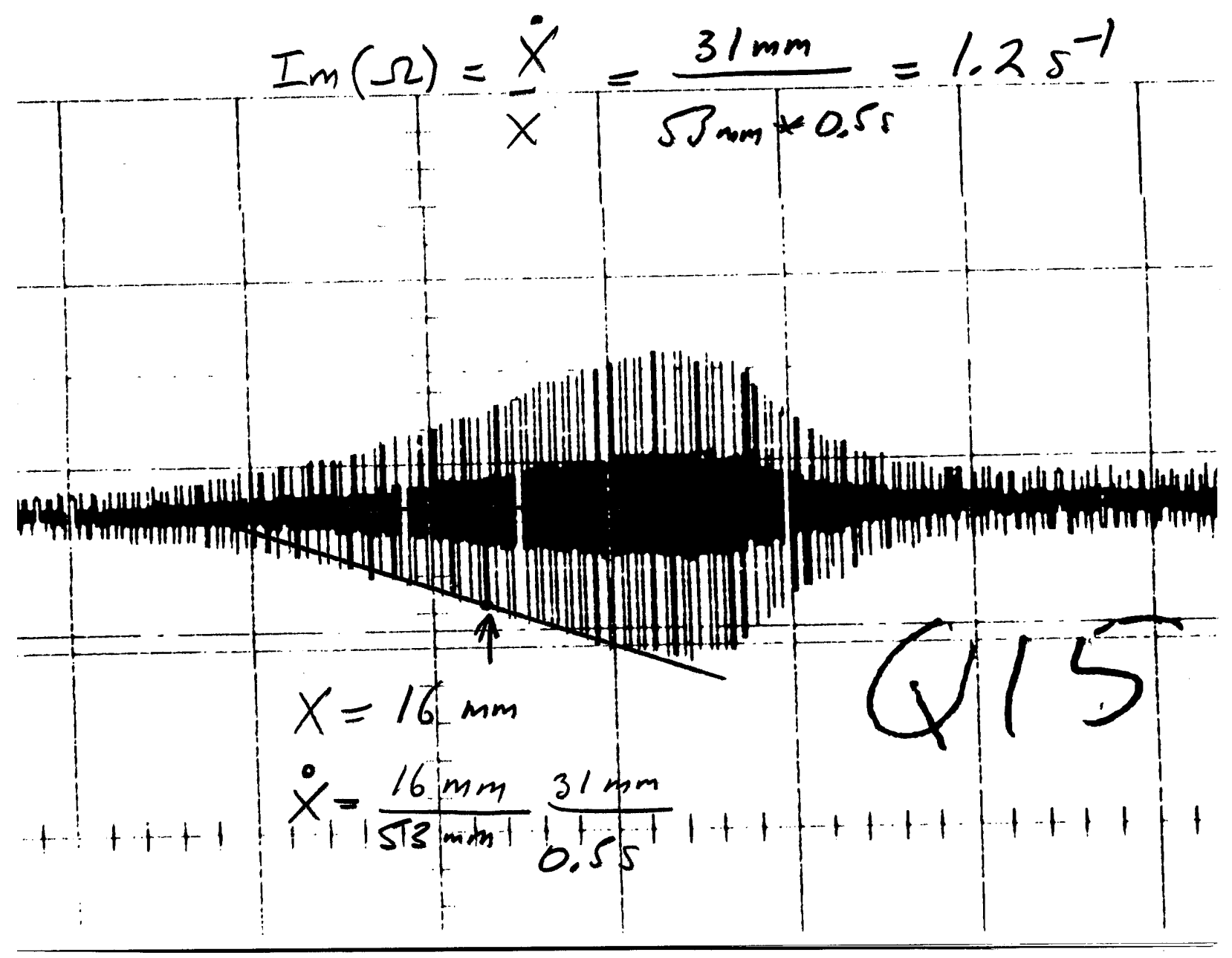

